VOLUME 30 (2021) 66-77

DOI: $10.24330 /$ ieja.969586

\title{
$f$-REPRESENTATIVES GROUPS
}

\author{
M. H. Hooshmand \\ Received: 8 December 2019; Revised: 12 October 2020; Accepted: 14 November 2020 \\ Communicated by Abdullah Harmancı
}

\begin{abstract}
The $b$-parts, $b$-addition of real numbers and some of their extensions (e.g., $f$-multiplications) were introduced and studied by the author. In this paper, we introduce $f$-representatives groups of a given group $(G, \cdot)$ which can be considered as a generalization of the group of the least non-negative residues (modulo $n$ ). Thereafter, we study some of their important properties and their relations among the ground group $(G, \cdot)$, a related quotient group, and $f$-grouplikes. Finally, many equivalent conditions for $f$-representatives groups and several examples in the group of real numbers are given.
\end{abstract}

Mathematics Subject Classification (2020): 20F99, 20M99

Keywords: $b$-Addition, $f$-multiplication, $f$-grouplike, decomposer function

\section{Introduction and preliminaries}

The term "Grouplike" was introduced and studied in [6]. Grouplike is an algebraic structure between semigroup and group and its axioms are generalization of the four group axioms. A homogroup is a semigroup that contains an ideal subgroup (see $[3,8,10]$ ). It is shown that a homogroup is a grouplike if and only if the set of its central idempotents is a singleton. Also, decomposer and associative functions on groups and semigroups were studied in 2007 and their general form were characterized on arbitrary groups. The topic has many applications for solving some functional equations on algebraic structures (see $[1,7]$ ). The $b$-decimal part function is a nice example of associative and strong decomposer functions and $\left(\mathbb{R},+_{b}\right)$ is a semigroup with some additional properties, where $+_{b}$ is $b$-addition. In fact $+_{b}$ is a type of $f$-multiplication and $\left(\mathbb{R},+_{b}\right)$ is a class united grouplike, where $f$ is the $b$-decimal part function (see $[6,7]$ ). In general, if $f$ is an associative function from a group $(G, \cdot)$ to itself, then we obtain the semigroup $\left(G, \cdot_{f}\right)$ that is a type of grouplikes (namely $f$-grouplike) where $\cdot_{f}$ is $f$-multiplication of the binary operation ".".

1.1. Decomposer, associative and canceler functions on groups. Let $(G, \cdot)$ be a group with the identity element $e$ and denote by $\iota_{G}$ the identity function on $G$. 
For every functions $f, g: G \rightarrow G$, consider the functions $f \cdot g$ and $f^{-}$defined by by $f \cdot g(x)=f(x) g(x), f^{-}(x)=f(x)^{-1}$. Also, the left (resp. right) $*$-conjugate of $f$ is given by $f^{*}=\iota_{G} \cdot f^{-}\left(\right.$resp. $\left.f_{*}=f^{-} \cdot \iota_{G}\right)$. Note that $G=f^{*}(G) f(G)=f(G) f_{*}(G)$ and the product is not necessarily direct (it is direct if $f$ is decomposer, see [7] and the next subsection).

If $(G,+)$ is an additive group, then the notations $e, f^{-}, f \cdot g, f \cdot g^{-}$should be replaced by $0,-f, f+g, f-g$ and we have $f^{*}=f_{*}=\iota_{G}-f$.

Example 1.1. Here, we recall the $b$-parts functions from [6] as some important examples in the topic. Fix a real number $b \neq 0$ and for every real number $a$, put $[a]_{b}:=b\left[\frac{a}{b}\right]$ (resp. $\left.(a)_{b}:=b\left\{\frac{a}{b}\right\}\right)$ that is called $b$-integer (resp. $b$-decimal) part of $a$ (where $\{t\}:=(t)=t-[t]$ is the fractional or decimal part of $t$ ). It is interesting to know that if $b>0$, then $(a)_{b}$ is the same remainder of the generalized division of $a$ by $b$ and this is our first idea for generalization of the least residues group $\bmod n$. Also, if $b$ is a positive integer, then $[a]_{b}$ is the same unique integer of the residue class $\{[a]-b+1, \cdots,[a]\}$ that is divisible by $b$.

The $b$-parts (real) functions ()$_{b}$ and []$_{b}$ have the properties ()$_{b}^{*}=[]_{b}$ and []$_{b}^{*}=()_{b}$, both are idempotent, so their compositions are zero and

$$
\begin{gathered}
\mathbb{R}_{b}:=(\mathbb{R})_{b}=\left\{(x)_{b} \mid x \in \mathbb{R}\right\}=b[0,1)=\{b d \mid 0 \leq d<1\}, \\
{[\mathbb{R}]_{b}=\left\{[x]_{b} \mid x \in \mathbb{R}\right\}=b \mathbb{Z}=\langle b\rangle .}
\end{gathered}
$$

For every function from $G$ to $G$ and $x, y \in G, f(x)=f(y)$ implies that $x=$ $f^{*}(x) f(y)=f(y) f_{*}(x)$. The converse is valid if $f$ is decomposer and we arrive at the following definition from [7].

Definition 1.2. A function $f$ from $G$ to $G$ is called

(a) right (resp. left) decomposer if

$$
f\left(f^{*}(x) f(y)\right)=f(y) \quad\left[\text { resp. } f\left(f(x) f_{*}(y)\right)=f(x)\right] \quad: \forall x, y \in G .
$$

(b) right (resp. left) strong decomposer if

$$
f\left(f^{*}(x) y\right)=f(y) \quad\left[\text { resp. } f\left(x f_{*}(y)\right)=f(x)\right] \quad: \forall x, y \in G .
$$

(c) right canceler (resp. left canceler) if

$$
f(x f(y))=f(x y)[\text { resp. } f(f(x) y)=f(x y)]: \quad \forall x, y \in G .
$$

(d) associative if

$$
f(x f(y z))=f(f(x y) z): \quad \forall x, y, z \in G .
$$


(e) strongly associative if

$$
f(x f(y z))=f(f(x y) z)=f(x y z): \quad \forall x, y, z \in G .
$$

Note that $f$ is decomposer (resp. canceler) if it is left and right decomposer (resp. canceler). In each parts of the above definition if $f(e)=e$, then we will add the word standard to the titles. Also, $f$ is standard right (resp. left) weak decomposer if and only if $f^{*} f=f f^{*}=e\left(\right.$ resp. $\left.f_{*} f=f f_{*}=e\right)$.

It is shown (see [7]) that every associative function is decomposer, every associative function with $f(e)=e$ is standard strong decomposer and strongly associative, and the following conditions are equivalent:

(i) $f$ is strongly associative,

(ii) $f$ is associative and idempotent,

(iii) $f$ is associative and $f^{2}(e)=f(e)$,

(iv) $f$ is associative and $f(e)$-periodic (i.e. $f(f(e) t)=f(t f(e))=f(t)$ for all $t$ ),

(v) $f$ is strong decomposer,

(vi) $f$ is decomposer and $f^{*}(G) \unlhd G$ or $f_{*}(G) \unlhd G$,

(vii) $f$ is decomposer and $f^{*}(G)=f_{*}(G) \unlhd G$,

(viii) $f$ is canceler.

Example 1.3. Fix a $b \neq 0$ in the additive group of real numbers. The $b$-decimal part function ()$_{b}$ satisfies all the above properties (i) till (viii) while the $b$-integer part function is only standard decomposer.

1.2. Direct product of subsets and their projections. Let $\Delta$ and $\Omega$ be subsets of a group $G$. By $\Delta \cdot \Omega$ we mean $G=\Delta \Omega$ and the product $G=\Delta \Omega$ is direct (i.e., $\delta_{1} \omega_{1}=\delta_{2} \omega_{2}$ and $\delta_{1}, \delta_{2} \in \Delta, \omega_{1}, \omega_{2} \in \Omega$ imply that $\delta_{1}=\delta_{2}$ and $\omega_{1}=\omega_{2}$ ). Also, we let $G=\Delta: \Omega$ denotes $G=\Delta \cdot \Omega$ and $\Delta \cap \Omega=\{e\}$. Note that additive notations are $\Delta \dot{+} \Omega$ (direct sum of subsets) and $\Delta \ddot{+} \Omega$ (standard direct sum of subsets). For example $\mathbb{R}=b \mathbb{Z} \ddot{+} b[0,1)=\langle b\rangle \ddot{+} \mathbb{R}_{b}$. If $\Delta$ and $\Omega$ are nonempty subsets of $G$, then $\Delta \Omega=\Delta \cdot \Omega$ if and only if $\left(\Delta^{-1} \Delta\right) \cap\left(\Omega \Omega^{-1}\right)=\{e\}$ (in additive notation $(\Delta-\Delta) \cap(\Omega-\Omega)=\{0\})$. Now, let $G=\Delta \cdot \Omega$ and define the functions $P_{\Delta}, P_{\Omega}: G \rightarrow G$, by $P_{\Delta}(x)=\delta$ and $P_{\Omega}(x)=\omega$ where $x=\delta \omega, \delta \in \Delta$ and $\omega \in \Omega$. Clearly, they are well-defined and $P_{\Delta}(G)=\Delta, P_{\Omega}(G)=\Omega, P_{\Omega}^{*}=P_{\Delta}$. We call $P_{\Omega}$, (resp. $\left.P_{\Delta}\right)$ right (resp. left) projection of the direct decomposition $G=\Delta \cdot \Omega$ (see [7]). The $b$-parts functions are projections of the direct decomposition $\mathbb{R}=\langle b\rangle \ddot{+} \mathbb{R}_{b}$. One can get more information about factorization of groups by their subsets from $[2,4,5,9,11]$. 


\section{2. $f$-Representatives groups}

If $f:(G, \cdot) \rightarrow(G, \cdot)$ is associative, then $(G, \cdot f)$ is a semigroup with some additional properties (namely $f$-grouplike), where $\cdot_{f}$ is the $f$-multiplication defined by $x \cdot f y=f(x y)$ for all $x, y \in G$. Also, according [7] one can drive that if $f$ is associative, then for every $x, y \in G$,

$$
\begin{array}{r}
f(f(x) y)=f(x f(y))=f(f(e) x y)=f(x f(e) y)=f(x y f(e))=f^{2}(x y) \\
f\left(f^{*}(x) y\right)=f\left(f^{*}(e) y\right)=f\left(y f^{*}(e)\right), f\left(x f_{*}(y)\right)=f\left(x f_{*}(e)\right)=f\left(f_{*}(e) x\right) .
\end{array}
$$

Now, we arrive at the following fundamental theorem that is the main key of the topic.

Theorem 2.1. If $f:(G, \cdot) \rightarrow(G, \cdot)$ is associative, then $(f(G), \cdot f)$ is a group with then following properties:

(a) It is the largest subgroup and also the least ideal of the semigroup $\left(G, \cdot_{f}\right)$.

(b) $f^{*}(G)=f_{*}(G)$ and $G=f^{*}(G) \cdot f(G)=f(G) \cdot f^{*}(G)$. So $f(G)$ is a factor subset of $(G, \cdot)$.

(c) Putting $g=f(e)^{-1} \cdot f, h=f \cdot f(e)^{-1}$ we have $f, g$ are standard strongly associative, strong decomposer and

$$
\begin{gathered}
g^{*}(G)=g_{*}(G)=h^{*}(G)=h_{*}(G)=f^{*}(G) f(e)=f(e) f^{*}(G) \unlhd(G, \cdot), \\
\frac{G}{f^{*}(G) f(e)} \cong\left(f^{-}(e) f(G), \cdot g\right) \cong\left(f(G) f^{-}(e), \cdot h\right) . \\
\text { Especially, if } f(e)=e, \text { then } f^{*}(G) \unlhd(G, \cdot) \text { and }
\end{gathered}
$$

$$
(f(G), \cdot f) \cong \frac{G}{f^{*}(G)} .
$$

(d)

$f$ is associative $\Rightarrow\left(f(G), \cdot_{f}\right)$ is a group with the identity $e_{f}:=f\left(f(e)^{-2}\right)$,

$f$ is strongly associative $\Rightarrow(f(G), \cdot f)$ is a group with the identity $f(e)$,

$f$ is associative and $f(e)=e \Rightarrow(f(G), \cdot f)$ is a group with the identity $e$.

Proof. Let $f:(G, \cdot) \rightarrow(G, \cdot)$ be associative.

(a) It is clear that $(G, \cdot f)$ is a semigroup with $(f(G), \cdot f)$ as a sub-semigroup. The element $\delta:=f\left(f(e)^{-2}\right)$ is an identity of $\left(f(G), \cdot_{f}\right)$, because by using the relations (1) and (2) we have

$$
\begin{gathered}
f(x) \cdot f \delta=f\left(f(x) f\left(f(e)^{-2}\right)\right)=f\left(f(x) f(e)^{-2} f(e)\right)=f\left(x f(e)^{-2} f(e)^{-2}\right) \\
=f(x)=\delta \cdot_{f} f(x) \quad: \quad \forall x \in G .
\end{gathered}
$$


Putting $w=f\left(f(e)^{-3} f(x)^{-1}\right) \in f(G)$ we have

$$
\begin{gathered}
f(x) \cdot f w= \\
=f\left(f(x) f\left(f(e)^{-3} f(x)^{-1}\right)=f\left(f(x) f(e) f(e)^{-3} f(x)^{-1}\right)\right. \\
=f\left(f(x) f(x)^{-1} f(e)^{-2}\right)=f\left(f(e)^{-2}\right)=\delta .
\end{gathered}
$$

Therefore, $(f(G), \cdot f)$ is a group. But, if $H$ is a sub-semigroup of $(G, \cdot f)$ with a left (resp. right) identity $e_{H}$, then $h=e_{H} \cdot f h=f\left(e_{H} h\right) \in f(G)$ (resp. $h=h \cdot \cdot_{f} e_{H}=$ $f\left(h e_{H}\right)$ ), for all $h \in H$, and so $\left(f(G), \cdot_{f}\right)$ is the largest subgroup of the semigroup $\left(G, \cdot_{f}\right)$. In addition, it is clear that $\left(f(G), \cdot_{f}\right)$ is an ideal of $\left(G, \cdot_{f}\right)$ and if $I$ is a left or right ideal and $a \in I$, then

$$
f\left(f(e)^{-2}\right)=a \cdot f f\left(f(e)^{-3} f(a)^{-1}\right)=f\left(f(e)^{-3} f(a)^{-1}\right) \cdot f a \in I
$$

and so $f(x)=f(x) \cdot f \delta=\delta \cdot f f(x) \in I$, for all $x \in G$. Thus, $\left(f(G), \cdot_{f}\right)$ is also the least ideal of the semigroup $(G, \cdot f)$.

(b) We claim that

$$
f^{*}(G)=\{\delta \in G \mid f(\delta f(\delta))=f(\delta)\}=\{\delta \in G \mid f(f(\delta) \delta)=f(\delta)\}=f_{*}(G) .
$$

For if $\delta=f^{*}(t)$, for some $t \in G$, then $f(\delta f(\delta))=f\left(f^{*}(t) f(\delta)\right)=f(\delta)$ (because every associative function is decomposer). Conversely, if $f(\delta f(\delta))=f(\delta)$, then

$$
\left.f^{*}(\delta f(\delta)) f(\delta f(\delta))=f^{*}(\delta f(\delta)) f(\delta) \Rightarrow \delta f(\delta)\right)=f^{*}(\delta f(\delta)) f(\delta),
$$

and so $\delta=f^{*}(\delta f(\delta)) \in f^{*}(G)$. Analogously, the third and last sets are equal and the identities $f(\delta f(\delta))=f(\delta f(e \delta))=f(f(\delta e) \delta)=f(f(\delta) \delta)$ imply that the second and third sets also are equal. Therefore, proof of (b) is complete.

(c) It is obvious that $g^{*}=f^{*} \cdot f(e)$ and $h_{*}=f(e) \cdot f_{*}$. But $g, h$ are standard strong decomposer and so $g^{*}(G)=g_{*}(G)=f^{*}(G) f(e) \unlhd(G, \cdot)$ and analogously for $h$. Since, $g^{*}(G)=f^{*}(G) f(e)$ is a normal subgroup of $G$, then $a f^{*}(G) f(e) a^{-1} \subseteq$ $f^{*}(G) f(e)$, for all $a$, and we conclude that $f^{*}(G) f(e)=f(e) f^{*}(G)$.

Finally, the map $g:(G, \cdot) \rightarrow\left(g(G)=f^{-}(e) f(G), \cdot g\right)$ is an epimorphism with kernel $g^{*}(G)=f^{*}(G) f(e)$, and the map $\rho:\left(f^{-}(e) f(G), \cdot g\right) \rightarrow\left(f(G) f^{-}(e), \cdot{ }_{h}\right)$, defined by $\rho\left(f^{-}(e) f(x)\right)=f(x) f^{-}(e)$, is an isomorphism.

(d) This part is concluded from (a) (note that if $f$ is strongly associative, then it is $f(e)$-periodic and so $\left.f\left(f(e)^{-2}\right)=f(e)\right)$.

The above theorem has several interesting algebraic aspects that is our motivation for the study. First, we arrive at the next definition.

Definition 2.2. Let $f:(G, \cdot) \rightarrow(G, \cdot)$ be associative. Then, we call the group $\left(f(G), \cdot_{f}\right)$ reference $f$-representatives group (because of the above theorem). Also. every its subgroup is called a $f$-representatives group (of $G$ ). 
We observe that if $f$ is associative and $f(e)=e$, then every $f$-representatives group is the same group of all "representatives" of a quotient group, by Theorem $2.1(\mathrm{c})$. For example, $\mathbb{Z}_{n}=\{0,1,2,3,4, \ldots, n-1\}$, the group of the least nonnegative residues modulo $n$ with the $n$-addition $+_{n}$ (see the next example), is a representatives group of the quotient group $\overline{\mathbb{Z}}_{n}=\{\overline{0}, \overline{1}, \overline{2}, \ldots, \overline{n-1}\}=\mathbb{Z} / n \mathbb{Z}$.

Example 2.3. For every fixed real number $b \neq 0$ and each $x, y \in \mathbb{R}$, define the binary operation $+_{b}$ by $x+{ }_{b} y:=(x+y)_{b}$. Since $+_{b}=+_{()_{b}}$ and ()$_{b}$ is standard strongly associative, then $\left(\mathbb{R}_{b},+_{b}\right)$ is a reference ()$_{b}$-representatives group with the identity element 0 that is the reference b-bounded group discussed in [6]. All subgroups of the reference $b$-bounded group $\left(\mathbb{R}_{b},+b\right)$ are $f$-representatives groups. If $n$ is an integer, then $\left(\mathbb{Z}_{n},+_{n}\right) \leq\left(\mathbb{R}_{n},+_{n}\right)$ and if $n \geq 1$, then it is the least nonnegative residues group modulo $n$.

Now, fix $\alpha \in \mathbb{R}$ and put $f(x)=\alpha+(x)_{1}$. Then $\left(\mathbb{R},+_{f}\right)$ is a grouplike and

$$
\left(f(\mathbb{R}),+_{f}\right)=\left(\alpha+[0,1),+_{f}\right)=\left([\alpha, \alpha+1),+_{f}\right)
$$

is a reference $f$-representatives group with the identity element $\alpha+(-2 \alpha)_{1}$. Also, $f$ is strongly associative if and only if $\alpha$ is an integer and

$2 \alpha$ is an integer $\Leftrightarrow\left([\alpha, \alpha+1),+_{f}\right)$ is a group with identity element $\alpha$,

$$
\alpha=0 \Leftrightarrow\left([\alpha, \alpha+1)=[0,1), \cdot_{f}\right) \text { is a group with the identity } 0 .
$$

Now, we study some important properties of $f$-decompositional groups. At first, we need to introduce many types of subsets of groups.

Definition 2.4. Let $\Delta, \Lambda$ and $\Omega$ be subsets of $G$ and $f: G \rightarrow G$. We say $\Omega$ is left (resp. right) $\Delta$-closed with respect to $\Lambda$ if for every $\lambda \in \Lambda$ there exists $\delta \in \Delta$ such that $\delta \lambda \in \Omega$ (resp. $\lambda \delta \in \Omega$ ). Now we call $\Omega$ :

(a) $\Delta$-symmetric if $\Omega$ is left $\Delta$-closed with respect to $\Omega^{-1}$.

(b) $\Delta$-multiplicative or $\Delta$-additive if $\Omega$ is left $\Delta$-closed with respect to $\Omega^{2}:=$ $\Omega \Omega$.

(c) Left $\Delta$-subtractive if $\Omega$ is left $\Delta$-closed with respect to $\Omega^{-1} \Omega$.

(d) Left $f$-subtractive if $f\left(\Omega^{-1} \Omega\right) \subseteq \Omega$.

(The right and two-sided cases are defined analogously.)

(e) $f$-multiplicative (resp. $f$-symmetric) if $f\left(\Omega^{2}\right) \subseteq \Omega$ (resp. $f\left(\Omega^{-1}\right) \subseteq \Omega$ ).

(f) $f$-invariant (resp. $f$-subset) if $f(\Omega) \subseteq \Omega$ (resp. $\Omega \subseteq f(G)$ ).

Remark 2.5. Considering the above definition the property $f(\Lambda) \subseteq \Omega$ is corresponded to the property " $\Omega$ is $\Delta$-closed with respect to $\Lambda$ " (where $\Delta$ is replaced by $f)$. 
If $\Delta=\{e\}$, then $S$ is $\Delta$-symmetric if and only if $\Omega=\Omega^{-1}$, i.e. $\Omega$ is symmetric, therefore we can write symmetric instance $\{e\}$-symmetric. Also $\Omega$ is $\{e\}$-multiplicative (resp. $\{e\}$-subtractive) if and only if $\Omega \Omega \subseteq \Omega$ (resp. $\Omega \Omega^{-1} \subseteq \Omega$ or equivalently $\Omega^{-1} \Omega \subseteq \Omega$ ), i.e. $\Omega \dot{\leq} G$ (resp. $\left.\Omega \leq G\right)$ if $\Omega \neq \emptyset$.

Every $\emptyset \neq \Omega \subseteq G$ is $G$-closed with respect to every $\Lambda \subseteq G$. If $e \in \Delta$, then $\Omega$ is $\Delta$-closed with respect to every $\Lambda \subseteq \Omega$. Also if $\Omega$ is $\Delta$-closed with respect to $\Lambda$ and $\Omega^{\prime} \supseteq \Omega, \Delta^{\prime} \supseteq \Delta$ and $\Lambda^{\prime} \subseteq \Lambda$, then $\Omega^{\prime}$ is $\Delta^{\prime}$-closed with respect to $\Lambda^{\prime}$.

Let $\Omega, \Delta$ be nonempty and $e \in \Lambda$. If $\Omega$ is $\Delta$-closed with respect to $\Lambda$, then $\Delta \cap \Omega \neq \emptyset$. Hence if $\Omega$ is $\Delta$-subtractive, then $\Delta \cap \Omega \neq \emptyset$.

If $\Omega$ is $f$-multiplicative and $e \in \Omega$, then $\Omega$ is $f$-invariant (because $\Omega \subseteq \Omega \Omega$ ). Also if $\Omega$ is $f$-subtractive and $e \in \Omega$, then $\Omega$ is $f$-invariant and $f$-symmetric (because $\left.\Omega \cup \Omega^{-1} \subseteq \Omega \Omega^{-1}\right)$.

Example 2.6. The set $\mathbb{R}_{1}=[0,1)$ is $\mathbb{Z}$-closed with respect to $\mathbb{R}$ but $\mathbb{Q}^{c}$ is not $\mathbb{Z}$-closed $\left(\mathbb{Q}\right.$-closed) with respect to $\mathbb{R}$. Fix positive integer $n$ and put $\mathbb{D}_{n}=$ $\left\{0, \frac{1}{n}, \cdots, \frac{n-1}{n}\right\}$, then $\mathbb{D}_{n}$ is ()$_{1}$-symmetric, ( $)_{1}$-additive and ( $)_{1}$ subtractive. The set $\mathbb{Z}_{n}$ for all $n \in \mathbb{Z} \backslash\{0\}$ is $n$-subtractive. The reference $b$-bounded set $\mathbb{R}_{b}$ is $\langle b\rangle$ additive, $\langle b\rangle$-symmetric and $\langle b\rangle$-subtractive (simply we write $b$-subtractive). The decimal set $D=\left[0, \frac{1}{3}\right) \cup\left(\frac{2}{3}, 1\right)$ is $\mathbb{Z}$-symmetric but it is not $\mathbb{Z}$-additive and $\mathbb{Z}$ subtractive. If $\alpha$ is irrational, then $\left\{(n \alpha)_{1} \mid n \in \mathbb{N}\right\}$ is $\mathbb{Z}$-additive but it is not $\mathbb{Z}$ symmetric and $\mathbb{Z}$-subtractive. The set $\{1\}$ is $\mathbb{N}$-subtractive but it is not $\mathbb{N}$-additive and $\mathbb{N}$-symmetric.

By $\Delta \dot{\leq} G$ we mean $\Delta$ is a sub-semigroup of the group $G$.

Theorem 2.7. Let $\emptyset \neq \Omega \subseteq G$.

(a) If $e \in \Omega$ or $\Delta \dot{\leq} G$, then

$\Omega$ is left (resp. right) $\Delta$-subtractive $\Rightarrow \Omega$ is left (resp. right) $\Delta$-symmetric.

(b) If $\Delta \dot{\unlhd} G$ (i.e. $\Delta \dot{\leq} G$ and $g \Delta=\Delta g$ : for all $g \in G$ ), then

$\Omega$ is $\Delta$-multiplicative and $\Delta$-stmmetric $\Rightarrow \Omega$ is $\Delta$-subtractive.

(The converse of this is not valid.)

(c) If $\Delta \unlhd G$, then

$\Omega$ is $\Delta$-subtractive $\Leftrightarrow \Omega$ is $\Delta$-multiplicative and $\Delta$-stmmetric.

Proof. (a) Let $\Delta \dot{\leq} G$ and $\Omega$ be left $\Delta$-subtractive. Choose $\delta_{0} \in \Delta \cap \Omega$ (note that $\Delta \cap \Omega \neq \emptyset)$ so for every $s \in \Omega$ there exists $\delta \in \Delta$ such that $\delta \delta_{0} s^{-1} \in \Omega$. Therefore $\Omega$ is left $\Delta$-symmetric, because $\delta \delta_{0} \in \Delta$. In the case $e \in \Omega$, left $\Delta$-subtractive implies 
left $\Delta$-symmetric clearly.

(b) Let $s_{1}, s_{2} \in \Omega$. There exists $\delta_{2} \in \Delta$ such that $\delta_{2} s_{2}^{-1} \in \Omega$. So there exists $\delta_{1} \in \Delta$ for which $\delta_{1} s_{1}\left(\delta_{2} s_{2}^{-1}\right)=\delta_{1} \delta_{2}^{\prime}\left(s_{1} s_{2}^{-1}\right) \in \Omega$, for some $\delta_{2}^{\prime} \in \Delta$. Therefore $\Omega$ is right $\Delta$-subtractive (because $\delta_{1} \delta_{2}^{\prime} \in \Delta$ ). Similarly it is left $\Delta$-subtractive.

The converse of this proposition is not valid, because $\{1\}$ is $\mathbb{N}$-subtractive but it is not $\mathbb{N}$-additive.

(c) Let $\Omega$ be $\Delta$-subtractive. Then $\Omega$ is $\Delta$-symmetric, by (a). Now if $s_{1}, s_{2} \in$ $\Omega$, then $\delta_{2} s_{2}^{-1} \in \Omega$ for some $\delta_{2} \in \Delta$. Therefore there exists $\delta_{1} \in \Delta$ such that $\delta_{1} s_{1}\left(\delta_{2} s_{2}^{-1}\right)^{-1}=\delta_{1} s_{1} s_{2} \delta_{2}^{-1}=\left(\delta_{1} \delta_{2}^{\prime}\right)\left(s_{1} s_{2}\right) \in \Omega$, for some $\delta_{2}^{\prime} \in \Delta$. Considering $\Delta \leq G$ and (b) this proof is complete.

Lemma 2.8. Let $f$ be right (resp. left) strong decomposer, $\Omega$ be $f$-set, $\Lambda \subseteq G$ and put $\Delta^{*}:=f^{*}(G)$ (resp. $\Delta_{*}:=f_{*}(G)$ ). Then

$\Omega$ is left $\Delta^{*}$-closed (resp. right $\Delta_{*}$-closed) with respect to $\Lambda \Leftrightarrow f(\Lambda) \subseteq \Omega$.

Especially, $\Omega$ is left $f$-subtractive (resp. $f$-multiplicative ; $f$-symmetric) if and only if it is left $\Delta^{*}$-subtractive (resp. $\Delta^{*}$-multiplicative ; $\Delta^{*}$-symmetric) (analogously for the right case).

Proof. Let $\Omega$ be left $\Delta$-closed with respect to $\Lambda$ and $\lambda \in \Lambda$, then $\delta \lambda \in \Omega$, for some $\delta \in \Delta$, so $f(\lambda)=f(\delta \lambda) \in f(\Omega)=\Omega$. Therefore $f(\Lambda) \subseteq \Omega$. Conversely, if $f(\Lambda) \subseteq \Omega$ and $\lambda \in \Lambda$, then putting $\delta=f^{*}(\lambda)^{-1}$ we have $\delta \in \Delta$ and $f(\lambda)=\delta \lambda \in \Omega$.

Now, we are ready to proof some important equivalent conditions for a $f$-set to be an $f$-representatives group whenever $f$ is strongly associative.

Theorem 2.9. Let $f$ be strongly associative and put $\Omega_{f}:=f(G), \Delta_{f}:=f^{*}(G)=$ $f_{*}(G)$. Then, for every $\Omega \subseteq \Omega_{f}$ the following statements are equivalent.

(a) $\left(\Omega, ._{f}\right) \leq\left(\Omega_{f}, ._{f}\right)$,

(b) $\Omega$ is f-subtractive,

(b') $\Omega$ is $\Delta_{f}$-subtractive,

(c) $\Omega$ is $f$-multiplicative and $f$-symmetric,

(c') $\Omega$ is $\Delta_{f}$-multiplicative and $\Delta_{f}$-symmetric,

(d) $\left(\Delta_{f} \Omega, \cdot\right) \leq(G, \cdot)$.

Proof. Since $f$ be strongly associative, then for every $\omega_{1}, \omega_{2} \in G \supseteq \Omega$ we have

$$
\omega_{1 \cdot{ }_{f}} \omega_{2}^{-1}=\omega_{1 \cdot{ }_{f}} f\left(\omega_{2}^{-1}\right)=f\left(\omega_{1} \omega_{2}^{-1}\right) .
$$

By using the above identities, Theorem 2.7 and Lemma 2.8 the parts (a), (b), (b'), (c), (c') are equivalent. Now let (d) holds and $\Delta=\Delta_{f}$. If $s_{1}, s_{2} \in \Omega$, then 
$s_{1}, s_{2} \in \Delta \Omega$ so $s_{1} s_{2}^{-1} \in \Delta \Omega$ and so $s_{1 \cdot{ }_{f}} f\left(s_{2}^{-1}\right)=f\left(s_{1} s_{2}^{-1}\right) \in f(\Delta \Omega)=f(\Omega)=\Omega$, therefore $\Omega \leq \Omega_{f}$. Also if (a) holds and $g_{1}, g_{2} \in \Delta \Omega$, then $g_{1}=\delta_{1} s_{1}, g_{2}=\delta_{2} s_{2}$, for some $\delta_{i} \in \Delta$ and $s_{i} \in \Omega$. So $g_{1} g_{2}^{-1}=\delta_{1} \delta^{\prime} f^{*}\left(s_{1} s_{2}^{-1}\right) f\left(s_{1} s_{2}^{-1}\right)$, for some $\delta^{\prime} \in \Delta$, thus $g_{1} g_{2}^{-1} \in \Delta \Omega$ (because $\Delta \leq G$ and $f\left(s_{1} s_{2}^{-1}\right)=s_{1 \cdot{ }_{f}} f\left(s_{2}^{-1}\right) \in \Omega$ ).

Example 2.10. Theorem 2.9 and Example 2.6 imply that $\left(\mathbb{D}_{n},+_{1}\right)$ is an $f$-representatives $\left(()_{1}\right.$-representatives) group that we call it $n$-decimal group.

Remark 2.11. If $f$ is strongly associative and $\Omega$ is a $f$-subtractive set, then $\Omega$ can be considered as the followings cases:

(i) An $f$-subset of $G$ with the properties $f$-symmetric and $f$-additive $(\Omega \subseteq$ $\left.\Omega_{f} \subseteq G\right)$.

(ii) A subgroup of $\Omega_{f}\left(\left(\Omega, \cdot_{f}\right) \leq\left(\Omega_{f}, \cdot_{f}\right)\right)$.

(iii) A factor of a subgroup of $G\left(\Delta_{f} \cdot \Omega=\Delta_{f} \Omega \leq(G, \cdot)\right)$.

Hence, we have the groups $\left(\Delta_{f}, \cdot\right) \unlhd\left(\Delta_{f} \Omega, \cdot\right) \leq(G, \cdot)$ and $\left(\Omega, \cdot \cdot_{f}\right) \leq\left(\Omega_{f}, \cdot \cdot_{f}\right)$. We show some other their relations in this section.

Now we want to see that when a strong decomposer function $f$ is endomorphism and what is the relation between $f^{*}(G) \cdot f(G)$ and $f^{*}(G) \times f(G)$ ? Answering this question we state the following theorem.

Theorem 2.12. Let $f:(G, \cdot) \rightarrow(G, \cdot)$ and $\Omega_{f}=f(G), \Delta_{f}=f^{*}(G)$.

(a) If all elements of $\Omega_{f}$ commutes with all elements of $\Delta_{f}$, then $f$ is endomorphism if and only if $f^{*}$ is endomorphism.

(b) If $f$ is right strong decomposer and $\Omega_{f} \dot{\leq}(G, \cdot)$ and $\omega \Delta_{f} \subseteq \Delta_{f} \omega$, for every $\omega \in \Omega_{f}$, then $f$ is endomorphism and strongly associative, $\Omega_{f} \leq(G, \cdot)$, $\Delta_{f} \unlhd G$. Moreover, if $\Omega_{f} \delta \subseteq \delta \Omega_{f}$, for every $\delta \in \Delta_{f}$, then $f^{*}$ is also an endomorphism, $\Omega_{f} \unlhd G$ and $G=\Delta_{f} \cdot \Omega_{f}=\Delta_{f} \dot{\times} \Omega_{f} \cong \Delta_{f} \times \Omega_{f}$ ( $\dot{\times}$ is inner direct product of subgroups).

Proof. The property $\omega \Delta_{f} \subseteq \Delta_{f} \omega$ implies for every $x, y \in G$, there exists $y^{\prime} \in G$ such that $f(x) f^{*}(y)=f^{*}\left(y^{\prime}\right) f(x)$ so

$$
\begin{gathered}
f(x y)=f\left(f^{*}(x) f(x) f^{*}(y) f(y)\right)=f\left(f^{*}(x) f^{*}\left(y^{\prime}\right) f(x) f(y)\right) \\
=f\left(f^{*}(z) f(w)\right)=f(w)=f(x) f(y) .
\end{gathered}
$$

Thus $\Omega_{f} \leq G$. On the other hand $\Delta_{f} \leq G$ and

$$
g \Delta_{f}=\delta \omega \Delta_{f} \subseteq \delta \Delta_{f} \omega=\Delta_{f} \delta \omega=\Delta_{f} g,
$$

for every $g \in G$, thus $\Delta_{f} \unlhd G$. Now if $\Omega_{f} \delta \subseteq \delta \Omega_{f}$, for every $\delta \in \Delta_{f}$, then $\Omega_{f} g \subseteq g \Omega_{f}$ similarly, for every $g \in G$, and so $\Omega_{f} \unlhd G$. So every element of $\Delta_{f}$ commutes with 
every elements of $\Omega_{f}$ (because $\Delta_{f} \unlhd G, \Omega_{f} \unlhd G$ and $G=\Delta_{f} . \Omega_{f}$ and so $G=\Delta_{f} \dot{\times} \Omega_{f}$ ). Finally

$$
f^{*}(x y)=x y f^{-}(x y)=x f^{*}(y) f^{-}(x)=x f^{-}(x) f^{*}(y)=f^{*}(x) f^{*}(y) .
$$

Now we remember some basic properties that we need for the next theorems. For every function $f: \mathcal{S} \rightarrow \mathcal{S}$ ( $\mathcal{S}$ is an arbitrary nonempty set) the following statements are equivalent:

(a) $f$ is idempotent,

(b) There exists a nonempty set $\Omega \subseteq \mathcal{S}$ such that $f: \mathcal{S} \rightarrow \Omega$ is onto and $f(\omega)=\omega$ for all $\omega \in \Omega$,

(c) $f(\mathcal{S}) \cap A \subseteq f(A)$, for all $A \subseteq \mathcal{S}$.

Clearly $A$ is $f$-set if and only if $A=f(B)$ for some $B \subseteq \mathcal{S}$. If $A$ is invariant under $f$, then $\left.f\right|_{A}$ is a function from $A$ to $A$. Now if $f$ is idempotent, then

i) $A$ is $f$-set if and only if $f(A)=A$.

ii) $A$ is invariant under $f$ if and only if $f(\mathcal{S}) \cap A=f(A)$.

In addition if $\mathcal{S}=G$ is a group and $H \leq G$, then

i) $H$ is invariant under $f$ if and only if it is invariant under $f^{*}$ (resp. $f_{*}$ ).

ii) If $H$ is invariant under $f$, then $H=f^{*}(H) f(H)$.

The above explanations imply the following lemma.

Lemma 2.13. Let $f:(G, \cdot) \rightarrow(G, \cdot)$ and $A \subseteq G$.

(a) If $f$ is standard right weak decomposer and $A$ is invariant under $f$ and $f^{*}$ and $f^{*}(A) f(A) \subseteq A$, then $A=\left(f^{*}(G) \cap A\right)(f(G) \cap A)$.

(b) If $f$ is standard right decomposer and $H \leq G$ is invariant under $f$ or $f^{*}$, then $H$ is standard $f^{*}$-decomposable (i.e. $H=f^{*}(H): f(H)$ ) and

$$
H=\left(\Delta_{f} \cap H\right):\left(\Omega_{f} \cap H\right)=\Delta_{H}: \Omega_{H}
$$

(where $\Delta_{H}:=\Delta_{f} \cap H$ and $\Omega_{H}:=\Omega_{f} \cap H$ ).

(c) If $f$ is standard right decomposer and $\Delta_{f} \subseteq H \leq G$ (resp. $\Omega_{f} \subseteq H \leq G$ ), then $H=\Delta_{f}: \Omega_{H}$ (resp. $\left.H=\Delta_{H}: \Omega_{f}\right)$.

Corollary 2.14. (Unique representation of subgroups by decomposer functions)

(a) If $G=\Delta_{f}: \Omega_{f}$, then every subgroup $H$ containing $\Delta_{f}$ (resp. $\Omega_{f}$ ) has the unique representation $H=\Delta_{f}: \Omega^{\prime}\left(\right.$ resp. $\left.H=\Delta^{\prime}: \Omega_{f}\right)$, where $\Omega^{\prime}$ is $f$-set and $\Delta^{\prime}$ is $f^{*}$-set.

(b) If $G=\langle g\rangle: \Omega_{g}\left(\Omega_{g}=\Omega_{f}\right)$ then every subgroup $H$ containing (the fix element) $g$ has the unique representation $H=\langle g\rangle: \Omega^{\prime}$, where $\Omega^{\prime}$ is $f$-set. 
Corollary 2.15. Let $G$ be an arbitrary group with the factorization $G=A \cdot B$. If $H$ is a subgroup of $G$ containing $A$, then $H=A \cdot(B \cap H)$. Therefore, $A$ is also a left factor of $H$.

Example 2.16. Every 1-periodic real set $A$ (i.e. $1+A=A$ ) has the unique direct representation $A=D \dot{+}$, where $D \subseteq[0,1)$. Moreover, $A \leq \mathbb{R}$ if and only if $D$ is subtractive ( $\mathbb{Z}$-subtractive). Also if $D$ runs over $2^{[0,1)}$, then every 1-periodic real set is gotten, exactly one time, from the above equation.

Theorem 2.17. If $f$ is associative and $f(e)=e$, then

(i) Every subgroup $H$ containing $\Delta_{f}$ has the unique representation $H=\Delta_{f}$ : $\Omega^{\prime}$, where $\Omega^{\prime}$ is an $f$-representatives group.

(ii) All subgroups of $G$ containing $\Delta_{f}$ and all $f$-representatives groups $\left(\Delta_{f}\right.$ subtractive sets) can be put in $1-1$ correspondence.

(iii) If $\Delta_{f} \subseteq H \leq G$, then $\left(\Delta_{f} \unlhd H\right)$

$$
\frac{H}{\Delta_{f}}=\frac{H}{f^{*}(H)} \approx f(H)=\Omega_{H}=\Omega_{f} \cap H .
$$

Proof. (i) and (ii): Let $\Delta_{f} \leq H \leq G$, then $H=\Delta_{f}: \Omega^{\prime}$, by Corollary 2.14. So $\Omega^{\prime} \leq \Omega_{f}$ is the unique $f$-representatives group in the representation. Conversely, if $\Omega^{\prime} \leq \Omega_{f}$, then $\Delta_{f} \subseteq \Delta_{f} \Omega^{\prime} \leq G$.

(iii): Since $H$ is invariant under $f^{*}$, then $H=\Delta_{f}: \Omega_{H}$ and $\left.f\right|_{H}$ is right strong decomposer function on the group $H$ dependent to this representation (i.e. $\left.f\right|_{H}=$ $\left.P_{\Omega_{H}}\right)$.

Corollary 2.18. If $\langle g\rangle \unlhd G, G=\langle g\rangle: \Omega_{g}$, then

(i) Every subgroup $H$ containing $g$ has the unique representation $H=\langle g\rangle: \Omega^{\prime}$, where $\Omega^{\prime}$ is a g-decompositional group, that means it is the $f$-representatives group and $\Delta_{f}=\langle g\rangle$.

(ii) All subgroups of $G$ containing the fixed element $g$ and all $g$-decompositional groups ( $\langle g\rangle$-subtractive sets) can be put in $1-1$ correspondence.

(iii) If $g \in H \leq G$, then

$$
\frac{H}{\langle g\rangle} \approx \Omega_{H}^{g}=\Omega_{g} \cap H .
$$

Example 2.19. For every subset $R$ of real numbers, put $(R)_{b}:=\left\{(r)_{b} \mid r \in R\right\}$ and $[R]_{b}:=\left\{[r]_{b} \mid r \in R\right\}$. If $R$ is a sub-group of $(\mathbb{R},+)$ containing $b$, then $(R)_{b}=R \cap \mathbb{R}_{b}$, $\frac{R}{\langle b\rangle} \cong \frac{1}{b}(R)_{b}=D^{b}$ and

$$
\frac{R}{\langle b\rangle}=\frac{R}{[R]_{b}} \cong(R)_{b}=R \cap \mathbb{R}_{b} \cong D^{b} .
$$


Specially if $1 \in R$, then $\frac{R}{\mathbb{Z}} \cong(R)_{1}=R \cap[0,1)$ and so $\frac{\mathbb{R}}{\mathbb{Z}} \cong\left([0,1),+_{1}\right)$.

Also, if $D$ is a decimal group, then $\frac{b \mathbb{Z}+b D}{b \mathbb{Z}} \cong D$.

Acknowledgement. The authors would like to thank the referee for the valuable suggestions and comments.

\section{References}

[1] J. Aczél and J. Dhombres, Functional Equations in Several Variables, Encyclopedia of Mathematics and its Applications, 31, Cambridge University Press, Cambridge, 1989.

[2] G. M. Bergman, A note on factorizations of finite groups, J. Iran. Math. Soc., $1(2)$ (2020), 157-161.

[3] A. H. Clifford and D. D. Miller, Semigroups having zeroid elements, Amer. J. Math., 70(1) (1948), 117-125.

[4] K. Corrádi and S. Szabó, Factoring certain infinite abelian groups by cyclic subsets, Pure Math. Appl. Ser. A, 2 (1992), 285-290.

[5] G. Hajós, Über einfache und mehrfache Bedeckung des n-dimensionalen Raumes mit einem Würfelgitter, Math. Z., 47 (1941), 427-467.

[6] M. H. Hooshmand, Grouplikes, Bull. Iranian Math. Soc., 39(1) (2013), 65-85.

[7] M. H. Hooshmand, New results for the multiplicative symmetric equation and related conjecture, Aequationes Math., 94(1) (2020), 123-136.

[8] R. P. Hunter, On the structure of homogroups with applications to the theory of compact connected semigroups, Fund. Math., 52 (1963), 69-102.

[9] A. D. Sands, The factorization of abelian groups II, Quart. J. Math. Oxford Ser. (2), 13 (1962), 45-54.

[10] S. Schwarz, Semigroups with a universally minimal left ideal, Acta Sci. Math. (Szeged), 52(1-2) (1988), 21-28.

[11] S. Szabó and A. D. Sands, Factoring Groups Into Subsets, Lecture Notes in Pure and Applied Mathematics, 257, CRC Press, Boca Raton, FL, 2009.

\section{H. Hooshmand}

Department of Mathematics

Shiraz Branch, Islamic Azad University

Shiraz, Iran

e-mail: hadi.hooshmand@gmail.com, hooshmand@iaushiraz.ac.ir 